10

\title{
Effect of Confinement Strength on the Conversion Efficiency of Strained Core-Shell Quantum Dot Solar Cell*
}

\author{
(C) Anupam Sahu and Dharmendra Kumar
}

Department of Electronics and Communication Engineering,

Madan Mohan Malaviya University of Technology,

Gorakhpur-273010, India

e-mail: anupam.or.sahu@gmail.com, dharmendra0127@gmail.com

Received April 06, 2020

Accepted July 01, 2020

Accepted July 07, 2020

In this paper, the conversion efficiency (CE) of core-shell quantum dot (CSQD) solar cell is investigated within weak and strong confinements strength, using detailed balance model. The weak and strong confinement strength in solar cell structure is modeled using $\mathrm{ZnTe} / \mathrm{ZnSe}$ and $\mathrm{PbS} / \mathrm{CdS} \mathrm{CSQD}$, respectively. Considering size-dependent strain results of CE of CSQD solar cell for varying core radius is plotted with and without considering multiple exciton generation (MEG), and the results show the improvement in CE with MEG, thus indicating its importance in the low-dimensional system. The numerical results demonstrate that for the same CSQD size, the solar cell with a stronger confinement strength achieves the higher CE in comparison to the weaker confinement. Also, the MEG significantly increases the CE of stronger confined CSQD solar cell. The results plotted are in good agreement with the literature.

Keywords: conversion efficiency, quantum dot, core-shell, solar cell, multiple exciton generation.

* Полный текст статьи опубликован в „Optics and Spectroscopy“ 2020 V. 128. N 10. 